\title{
Serum KL-6 as a Possible Marker for Amiodarone-induced Pulmonary Toxicity
}

\author{
Masamitsu Nakajima, Yousuke Kawahara*, Kouichirou Yoshida, Naoyuki MiYashita, \\ Yoshihito NIKI and Toshiharu MATsUSHIMA
}

\begin{abstract}
Amiodarone is a useful drug for the treatment of lifethreatening cardiac arrhythmias. However, amiodarone can induced pulmonary toxicity (APT) and may cause lifethreatening lung damage. APT can be difficult to diagnose, but early diagnosis is important. Here, in a 51-year-old man with APT, the high serum KL-6 level was correlated with the severity of symptoms and chest $\mathrm{X}$-ray findings, and it was inversely correlated with $\mathrm{PaO}_{2}$ and diffusion capacity for carbon monoxide levels. The findings suggest that the serum KL-6 level may be increased in APT and that therefore it's the determination of serum KL-6 may provide a useful indicator and/or monitoring marker of APT. KL-6 is believed to be produced and secreted by type II pneumocytes. Typical pathological findings of APT include proliferation of type II pneumocytes which may produce KL-6, and result in increased serum KL-6 levels.

(Internal Medicine 39: 1097-1100, 2000)
\end{abstract}

Key words: drug induced-pneumonia, MUC-1, type II pneumocyte

\section{Introduction}

Amiodarone can cause drug-induced pulmonary toxicity; the incidence of toxicity ranges from 0.8 to $17 \%$, and it has life-threatening adverse effects (1-3). Early diagnosis of amiodarone-induced pulmonary toxicity (APT) is important. However, there are no characteristic symptoms, signs, or specific findings on laboratory data or chest radiography. Previously, we reported that the serum KL-6 level is a useful marker in drug-induced pneumonia (4). Herein we describe the serum KL-6 levels in an APT patient to demonstrate its usefulness as a marker of APT.
For editorial comment, see p 1004.

\section{Case Report}

A 51-year-old man with exertional dyspnea and low grade fever $\left(37^{\circ} \mathrm{C}\right)$ had been admitted to another hospital two weeks earlier, and was referred to our hospital on December 6, 1998. He had been diagnosed as having dilated cardiomyopathy with premature ventricular contraction (PVC). PVC had been well medicated, using amiodarone $(200 \mathrm{mg} /$ day) since November 1994. Chest X-ray films and a chest CT scan on admission revealed non-segmental diffuse ground glass opacities with peripheral predominance in both lung fields (Figs. 1,2). There was no purulent sputum. The patient indicated that he neither drank alcohol nor smoked. Physical examination on admission was unremarkable except for fine crackles in both lungs on chest auscultation. His arterial blood gas values were $\mathrm{pH} 7.45$, $\mathrm{PaO}_{2} 72 \mathrm{mmHg}, \mathrm{PaCO}_{2} 38 \mathrm{mmHg}$ and $\mathrm{A}-\mathrm{aDO}_{2}$ of 31 while breathing room air. The diffusion capacity for carbon monoxide $\left(\mathrm{D}_{\mathrm{LCO}}\right)$ was $11.10 \mathrm{ml} / \mathrm{min} / \mathrm{mmHg}(46.5 \%)$, inspiratory vital capacity (IVC) was $3.57 l(96.2 \%)$ and forced expiratory volume in $1 \mathrm{~s}\left(\mathrm{FEV}_{1}\right)$ was $2.72 l(77.5 \%)$ on pulmonary function testings. Gallium-67 scintigraphy showed abnormal lung uptake in both lungs. Other laboratory data revealed hemoglobin of $14.4 \mathrm{~g} / \mathrm{dl}$, a white blood cell count of $10,700 / \mu \mathrm{l}$ (neutrophils: $77.0 \%$; eosinophils: $2.0 \%$; lymphocytes: $17.0 \%$; monocytes: $4.0 \%$ ), an erythrocyte sedimentation rate of $44 \mathrm{~mm} / \mathrm{h}$ and C-reactive protein (CRP) of $1.9 \mathrm{mg} / \mathrm{dl}$. Blood chemical analysis disclosed a high serum lactate dehydrogenase (LDH) level of 1,032 IU/l. The lack of purulent sputum, high fever, and the findings on chest X-ray and pulmonary function test suggested that he had APT rather than bacterial pneumonia. We discontinued the amiodarone treatment and subsequently the dyspnea, low grade fever, the abnormal findings on chest $\mathrm{X}$-ray (Fig. 3), and gallium-67 scintigraphy, and abnormal laboratory data (serum $\mathrm{CRP}$ and $\mathrm{LDH}$ ), and $\mathrm{PaO}_{2}$ levels improved

From Division of the Respiratory Diseases and *Division of the Cardiology, the Department of Medicine, Kawasaki Medical School, Kurashiki Received for publication April 11, 2000: Accepted for publication July 20, 2000

Reprint requests should be addressed to Dr. Masamitsu Nakajima, the Division of Respiratory Diseases, the Department of Medicine, Kawasaki Medical School, 577 Matsushima, Kurashiki 701-0192 


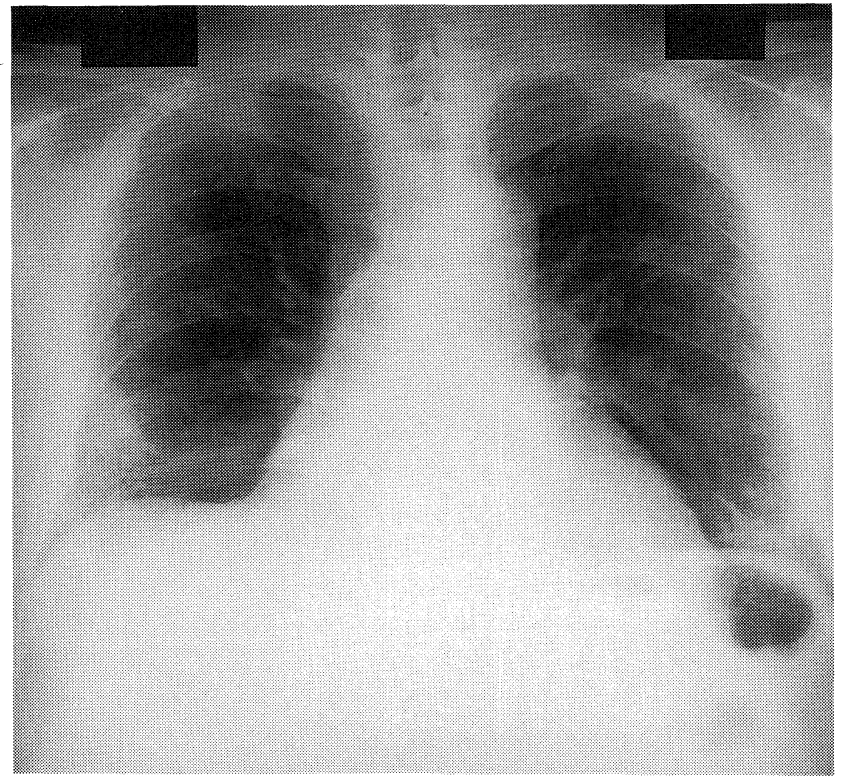

Figure 1. A chest X-ray film on admission reveals an infiltrative shadow and ground-glass opacities in both lower lung fields with peripheral predominance and cardiac dilatation.

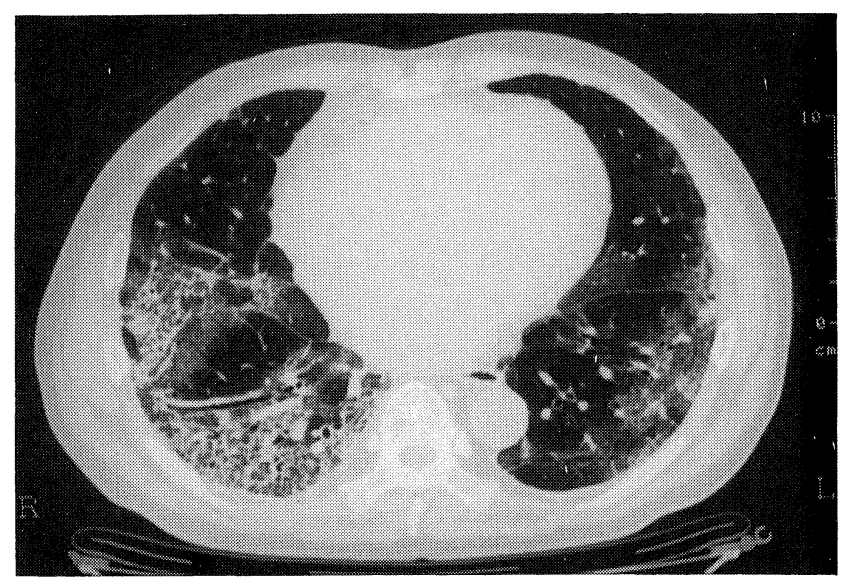

Figure 2. A chest CT scan on admission shows inter- and intra- lobular septal thickening, and ground-glass opacities in both lungs.

gradually. Serum precipitins against Trichosporon asahii, Trichosporon mucoides, and Aspergillus, Cryptococcus which are well known to be causative agents of hypersensitivity pneumonitis (HP) were negative. Serum antibody titers of Mycoplasma pneumoniae (CF), Chlamydia psittaci (immunofluorescence antibody technique: MFA), Chlamydia pneumoniae (MFA) and viruses such as influenzae virus A (HI), influenzae virus $B(\mathrm{HI})$, adenovirus (NT), and respiratory syncytial virus (CF) were negative.

KL-6 levels were examined with a sandwich-type enzyme-

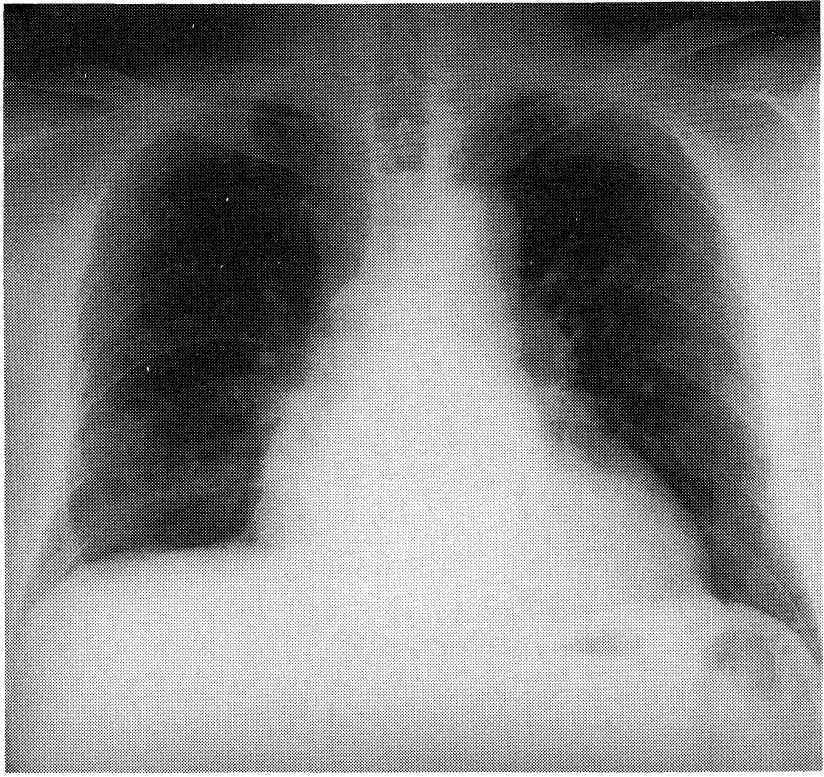

Figure 3. The infiltrative shadow and ground-glass opacities on chest X-ray improved on January 10, 1999.

linked immunosorbent assay (ELISA) using KL-6 monoclonal antibody (IgG1) in sera. The levels were high $(988 \mathrm{U} / \mathrm{ml}$ before discontinuing the amiodarone treatment) but gradually decreased to within the normal ranges after cessation of the amiodarone treatment. Serum KL-6 levels were related with the symptoms, opacities on the chest X-ray films, and abnormal shadows on gallium-67 scintigraphy and reversely correlated with the patient's $\mathrm{PaO}_{2}$ levels and $\mathrm{D}_{\mathrm{LCO}}$ levels (Fig. 4).

\section{Discussion}

Amiodarone was first introduced in 1967 as an antiangina agent, and was subsequently found to have potent antiarrhythmic properities. Initial reports emphasized its remarkable clinical efficacy in a variety of cardiac arrhythmias. With further experience, however, numerous adverse effects have become well recognized, of which pulmonary toxicity remains the most serious because of its potentially life-threatening nature. The reported incidence of APT has been highly variable (between 0.8 and $17 \%$ ), and it can be fatal (1-3). APT may involve fever, chest pain, dyspnea and alveolar or mixed alveolar-interstitial infiltrates on the chest X-ray. These symptoms and shadows on chest X-rays mimic those of pulmonary infections. Laboratory data are nonspecific, including an elevated erythrocyte sedimentation rate and frequent leukocytosis. Bronchoalveolar lavage (BAL) and biopsy are useful to rule out malignancy and infection. The inclusion-bearing macrophage can be identified in BAL specimens, supporting a diagnosis of APT (5). As indicated previously, however, such macrophages can also be seen in patients without evidence of disease and this finding is not diagnostic of APT-related pulmo- 


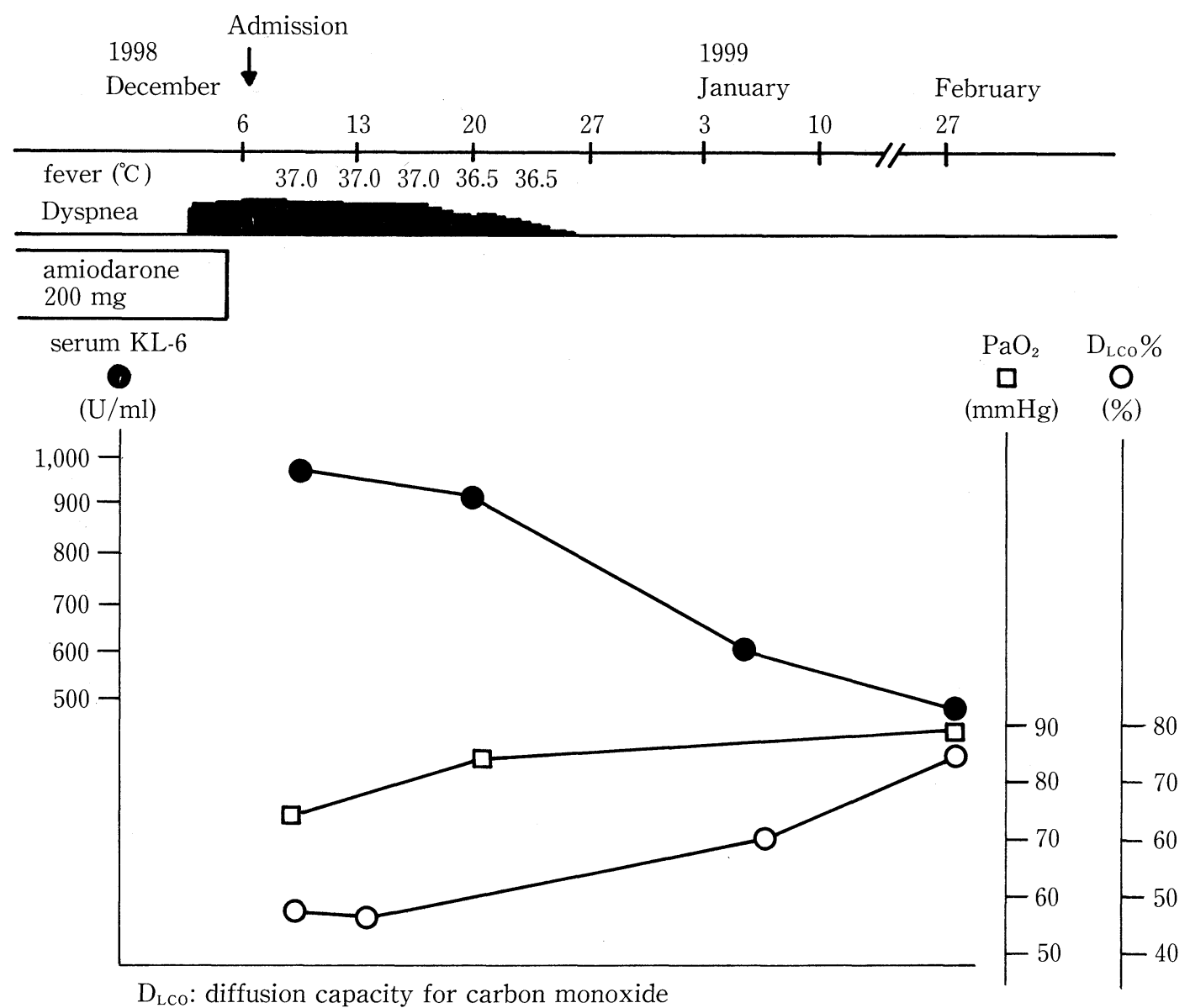

Figure 4. Clinical course. Cut-off levels of $\mathrm{KL}-6, \mathrm{PaO}_{2}$ and $\mathrm{LDH}$ are $500 \mathrm{U} / \mathrm{ml}, 80 \mathrm{mmHg}$ and $125 \mathrm{IU} / \mathrm{ml}$, respectively. The serum KL-6 level was related to the symptoms and inversely related to the patient's $\mathrm{PaO}_{2}$ levels and D $_{\text {LCo }}$ levels.

nary tissue damage (6). The diagnosis of APT remains one of exclusion and requires careful function and radiological investigations, and findings of BAL and lung biopsy.

We diagnosed this case as APT, because the patient had taken amiodarone for four years and we could not detect any organisms in the sputum culture or any high serum antibody titer of viruses, Mycoplasma pneumoniae, Chlamydia pneumoniae, or Chlamydia psittaci. A pulmonary function test disclosed a decrease in $\mathrm{D}_{\mathrm{LCO}}(46.5 \%)$, which was a decrease by more than $20 \%$ from the pre-treatment value. Gallium-67 scintigraphy revealed an abnormal uptake in the lungs. After discontinuation of the amiodarone treatment without steroid therapy, his symptoms, and abnormal laboratory data including pulmonary function test and uptake of gallium-67 in the lung and abnormal findings of chest X-ray improved.

Early diagnosis of APT is difficult, as it relies upon nonspecific symptoms, signs, radiological findings and abnormalities in pulmonary function tests, and laboratory data. However, early diagnosis is important to the treatment. Radiologic features of APT are characteristic but non-specific. A decrease in carbon monoxide lung diffusing capacity $\left(\mathrm{D}_{\mathrm{LCO}}\right)$ is a sensitive indicator of APT in the presence of symptoms and radiological findings; however, it lacks specificity. Asymptomatic patients taking amiodarone may show decreases reductions in $D_{\mathrm{LCO}}$ (7). Furthermore, diagnostic difficulties occur when the non-specific symptoms of APT are masked by the symptoms of cardiac failure in critically ill patients, with the diagnosis of APT being determined only after treatment of the underlying cardiac disease.

The serum KL-6 level is high in pulmonary fibrosis $(8,9)$ and we reported that the serum KL-6 level is high in druginduced pneumonia and hypersensitivity pneumonitis $(4,10)$. On the other hand, the serum KL-6 level is low in bacterial pneumonia, Mycoplasmal pneumonia, Chlamydia psittaci pneumonia and Chlamydia pneumoniae pneumonia (10). For these reasons, we examined the serum KL-6 levels in this patient with APT, and found that the serum KL-6 levels were related to the symptoms and findings on chest $\mathrm{X}$-ray and gallium-67 scintigraphy and the KL-6 levels were inversely related to $\mathrm{PaO}_{2}$ and $\mathrm{D}_{\mathrm{LCO}}$ levels. We think that serum KL- 6 could 


\section{NAKAJIMA et al}

be a useful serum marker in APT.

KL-6, a mucin-like glycoprotein that is now classified as cluster 9 (MUC-1) (11), is believed to be produced from the proliferation of type II pneumocytes (12). We could not clarify the mechanism of the high serum KL-6 levels in APT. However, the pathological features of APT may show type II pneumocyte proliferation, lymphocytic alveolitis, and in the late stage, alveolar and septal fibrosis $(6,13)$. We think that this proliferation of type II cells produces the KL-6. Further study is needed to clarify the mechanisms of KL-6 production from type II pneumocytes in APT.

Acknowledgements: This study was supported in part by a Research Project Grant (No. 12-410) from Kawasaki Medical School.

\section{Reference}

1) Dusman RE, Stanton MS, Miles WM, et al. Clinical features of amiodarone-induced pulmonary toxicity. Circulation. 82: 51-59, 1990.

2) Pollak PT. Clinical organ toxicity of antiarrhythmic compounds: ocular and pulmonary manifestations. Am J Cardiol 84: 37R-45R, 1999.

3) Jessurun GA, Crijns HJ. Amiodarone pulmonary toxicity. BMJ 314: 619$620,1997$.

4) Nakajima M, Manabe T, Mitekura H, Hashiguchi K, Niki Y, Matsushima T. Levels of serum KL-6 in a patient with drug-Induced Pneumonitis.
Nihon-Kyobu-Shikkan-Gakkai-Zasshi 35: 813-817, 1997 (in Japanese with English Abstract)

5) Liu FL, Cohen RD, Downar E, Butany JW, Edelson JD, Rebuck AS Amiodarone pulmonary toxicity: functional and ultrastructural evaluation. Thorax 41: 100-105, 1986.

6) Myers JL, Kennedy JI, Plumb VJ. Amiodarone lung: pathologic findings in clinically toxic patients. Hum Pathol 18:349-359, 1987.

7) Gleadhill IC, Wise RA, Schonfeld SA, et al. Serial lung function testing in patients treated with amiodarone: a prospective study. Am J Med 86: 4-10, 1989.

8) Kohno N, Koizumi S, Awaya Y, Fukuhara H, Yamakido M, Akiyama M. New serum indicator of interstitial pneumonitis activity: sialylated carbohydrate antigen KL-6. Chest 96: 68-73, 1989.

9) Kobayashi J, Kitamura S. KL-6: a serum marker for interstitial pneumonia. Chest 108: 311-315, 1995.

10) Nakajima M, Toshiaki M, Yoshida K, Niki Y, Matsushima T. Evaluation of serum KL-6 levels in summer-type hypersensitivity pneumonitis. Nihon-Kokyuki-Gakkai-Zasshi 36: 763-770, 1998 (in Japanese with English Abstract).

11) Kohno N, Inoue $Y$, Hamada $H$, et al. Difference in sero-diagnostic values among KL-6-associated mucins classified as cluster 9. Int J Cancer Suppl 8: 81-83, 1994.

12) Nakajima M, Manabe T, Niki Y, Matsushima T. Serum Kl-6 level as a monitoring marker in a patient with pulmonary alveolar proteinosis. Thorax 53: 809-811, 1998 .

13) Kennedy JI, Myers JL, Plumb VJ, Fulmer JD. Amiodarone pulmonary toxicity. Clinical, radiologic, and pathologic correlations. Arch Intern Med 147: 50-55, 1987. 